\title{
Recurrence-free survival following aggressive multimodal treatment of an isolated recurrence of adrenocortical carcinoma
}

\author{
Alexander Magony ${ }^{1,2(i)}$, Adam Mutsaers ${ }^{3}$ (i) \& Belal Ahmad*, Ad $^{*}$ \\ ${ }^{1}$ Schulich School of Medicine, Western University, London, Ontario N6A 5C1, Canada \\ ${ }^{2}$ Division of General Internal Medicine, Department of Medicine, University of Toronto, Toronto, Ontario M5S 3H2, Canada \\ ${ }^{3}$ Department of Radiation Oncology, London Regional Cancer Program, London, Ontario N6A 5W9, Canada \\ *Author for correspondence: Belal.Ahmad@Ihsc.on.ca
}

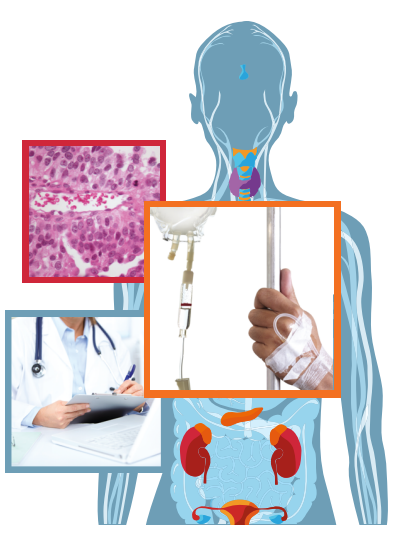

International Journal of Endocrine Oncology

\begin{abstract}
A 43-year old woman demonstrated an intra-abdominal drop metastasis 6 years after initial presentation of stage-III adrenocortical carcinoma (ACC), previously treated with resection, adjuvant radiation and mitotane therapy. This recurrence was managed aggressively with complete excision, adjuvant radiation and mitotane. Imaging at 3.25-year follow-up showed no evidence of recurrence, a remarkable response given ACC's poor prognosis and high recurrence rates. Management of oligometastatic ACC remains unclear and intra-abdominal drop metastases are particularly rare in ACC; both require further documentation. Aggressive management with adjuvant radiation and mitotane therapy may represent an effective and welltolerated approach for improving local control for recurrent ACC, including drop metastases. Further research is required to codify potential benefits.
\end{abstract}

Tweetable abstract: A 43-year old woman with drop metastasis 6 years after primary adrenocortical carcinoma resection received re-resection with adjuvant therapy and is recurrence-free 3.25 years later. This may be an effective approach for local control in recurrent adrenocortical carcinoma, which is poorly understood.

First draft submitted: 10 September 2020; Accepted for publication: 6 January 2021; Published online: 31 March 2021

Keywords: ACC $\bullet$ adjuvant therapy $\bullet$ adrenal $\bullet$ adrenocortical carcinoma $\bullet$ cancer $\bullet$ metastasis $\bullet$ mitotane $\bullet$ radiation therapy $\bullet$ recurrence-free survival $\bullet$ salvage therapy

Adrenocortical carcinoma (ACC) is a rare cancer of the adrenal cortex that carries a poor prognosis, with an incidence of 1 to 2 cases per million per year and a 5-year survival of 25-40\% [1]. Surgical resection represents the only established curative option for ACC [2] and the preferred treatment for both primary tumors and isolated metastases [3]. There are few established options to improve local control and survival in the adjuvant setting. Even after initial complete resection, recurrence occurs in up to $80 \%$ of patients [4], contributing to low survival rates [3]. The use of mitotane chemotherapy has led to improvements in recurrence-free survival time in some patients [5,6], while combined chemotherapy and mitotane has shown promise as well [1]. Adjuvant radiation with standard fractionation following resection may improve local recurrence rates, though no overall survival benefit has been demonstrated [7].

Given the high rates of recurrence, salvage therapy options are important but remain currently underinvestigated. In patients with recurrent ACC, the benefits of repeat resection are contested, though, may be indicated in select patients who meet favorable criteria [8]. The utility of adjuvant mitotane or chemotherapy following resection of metastatic or recurrent disease remains unclear [8]. A recent study demonstrates that hyperthermic intraperitoneal chemotherapy immediately following primary resection has been shown to improve local recurrence, though is limited by significant morbidity and requires careful candidate selection. Furthermore, it has not yet demonstrated clear benefit in treating recurrent disease [9]. In patients with unresectable locally advanced or metastatic disease, Laganà et al. (2020) have shown some long-term benefit in EDP-M chemotherapy (etoposide, doxorubicin and 
Table 1. Relevant case reports of recurrent adrenocortical carcinoma involving abdominal metastases.

\begin{tabular}{|c|c|c|c|c|}
\hline Study (year) & Summary & Treatment & Outcome & Ref. \\
\hline Current case & $\begin{array}{l}\text { A } 4.5 \times 3.5 \times 3.0-\mathrm{cm} \text { pelvic sidewall metastasis outside of } \\
\text { previous resection and RT field, } 6 \text { years post-treatment } \\
\text { (open right adrenalectomy and adjuvant mitotane and } \\
\text { radiation therapy) }\end{array}$ & $\begin{array}{l}\text { Metastasis treated with complete } \\
\text { open excision as well as adjuvant } \\
\text { radiotherapy }(22.5 \mathrm{~Gy} / 5) \text { and } \\
\text { mitotane }\end{array}$ & $\begin{array}{l}\text { Well tolerated and no evidence of } \\
\text { recurrence at } 18 \text { months } \\
\text { postradiotherapy }\end{array}$ & \\
\hline Bhatkule et al. (2015) & $\begin{array}{l}\text { Initial presentation of a } 10 \times 7 \times 4-\mathrm{cm} \text { ACC tumor with a } \\
0.5 \times 0.4-\mathrm{cm} \text { abdominal wall metastasis }\end{array}$ & Treatment not documented & Outcome not documented & [21] \\
\hline Gupta et al. (2012) & $\begin{array}{l}\text { A } 6.5 \times 6.6 \times 5.4-\mathrm{cm} \text { abdominal wall scar metastasis } \\
\text { presenting } 1.5 \text { years following open resection of the } \\
\text { primary tumor with the left kidney, which occurred } \\
\text { without rupture of the capsule or spillage of the tumor } \\
\text { cells }\end{array}$ & $\begin{array}{l}\text { Surgical resection was refused in } \\
\text { favor of traditional medicines }\end{array}$ & $\begin{array}{l}\text { The patient presented } 2 \text { months } \\
\text { later suffering from profuse } \\
\text { bleeding and shock before } \\
\text { deceasing }\end{array}$ & [19] \\
\hline Morin et al. (2012) & $\begin{array}{l}\text { A } 2.2 \times 2.2-\mathrm{cm} \text { recurrence in the tumor bed, } 3 \text { months } \\
\text { after the initial surgery (laparoscopic right } \\
\text { adrenalectomy). The recurrence was excised with the } \\
\text { right kidney and adjacent lymph nodes with negative } \\
\text { margins. The patient remained clear of disease for } \\
29 \text { months until it metastasized to the liver }\end{array}$ & $\begin{array}{l}\text { To treat the liver metastasis, the } \\
\text { patient received chemotherapy } \\
\text { (cisplatin, etoposide and } \\
\text { doxorubicin), hepatic stereotactic } \\
\text { external-beam radiotherapy } \\
\text { ( } 42 \mathrm{~Gy} / 6 \text { ) and multikinase inhibitor } \\
\text { sorafenib }\end{array}$ & $\begin{array}{l}\text { Well tolerated with a partial } \\
\text { response as the liver metastasis } \\
\text { reduced in size and the right adrenal } \\
\text { bed remained clear. However, her } \\
\text { disease continued to spread to her } \\
\text { lungs }\end{array}$ & [22] \\
\hline $\begin{array}{l}\text { Van Grevenstein } \\
\text { et al. (2001) }\end{array}$ & $\begin{array}{l}\text { A } 5-\mathrm{cm} \text { abdominal wall metastasis detected in the } \\
\text { surgical scar } 4.5 \text { years following open resection of the } \\
\text { primary tumor, which was complicated by capsule } \\
\text { rupture and tumor spill }\end{array}$ & $\begin{array}{l}\text { The metastasis was resected radically } \\
\text { with negative margins but vascular } \\
\text { invasion. No adjuvant therapy was } \\
\text { given }\end{array}$ & $\begin{array}{l}\text { No recurrence as of } 12 \text {-month } \\
\text { follow-up }\end{array}$ & [20] \\
\hline Foxius et al. (1999) & $\begin{array}{l}\text { Extensive peritoneal carcinomatosis confirmed via } \\
\text { laparotomy } 11 \text { months after a laparoscopic excision of } \\
\text { what was originally considered a 2-cm Conn's adenoma }\end{array}$ & $\begin{array}{l}\text { The ACC recurrence was debulked } \\
\text { and treated with adjuvant mitotane }\end{array}$ & $\begin{array}{l}\text { The patient deceased } 9 \text { months after } \\
\text { the debulking procedure }\end{array}$ & [23] \\
\hline Hamoir et al. (1998) & $\begin{array}{l}\text { Extensive peritoneal recurrence } 6 \text { months after } \\
\text { laparoscopic excision of a } 12-\mathrm{cm} \text { ruptured adrenocortical } \\
\text { carcinoma }\end{array}$ & $\begin{array}{l}\text { The recurrence was debulked and } \\
\text { then treated with intraperitoneal } \\
\text { cisplatinum chemotherapy as well as } \\
\text { six courses of systemic chemotherapy } \\
\text { (cisplatinum, etoposide and } \\
\text { mitotane) }\end{array}$ & Remission & [24] \\
\hline
\end{tabular}

cisplatin plus oral mitotane), including the eventual possibility of surgically excising residual disease following treatment response [10]; however, this similarly comes at the cost of systemic chemotherapy with significant toxicity.

There remains tremendous value in elucidating the role of well-tolerated modalities in treating recurrent disease as alternative treatments such as hypofractionated radiotherapy, radiofrequency ablation, microwave ablation and cryoablation may represent opportunities to preserve quality of life and reduce surgery-associated morbidity [11]. Though modern immunotherapy now plays a major role in the treatment of other cancers, it has yet to demonstrate a clear role in the management of ACC [10]. Recent studies suggest that biologic agents, such as pembrolizumab, may offer a favorable toxicity profile and modest clinical benefit in the treatment of ACC, which may be further characterized in future studies [12].

Conventionally fractionated external beam radiotherapy has been given postoperatively at the adrenalectomy site of advanced primary tumors to mixed results [13-15]. Radiation has also previously been used palliatively to help control symptoms in late-stage ACC, though no survival benefit has been demonstrated [1,16]. Stereotactic radiotherapy has not been formally investigated as a treatment for unresectable tumors but may be beneficial in well-performing patients with limited metastases to the brain, lungs or liver $[17,18]$.

Isolated peritoneal ACC metastases in a postoperative setting with known tumor spill is a rare but known presentation of recurrence [11]. Abdominal wall metastases have been described in case reports summarized in Table $1[19,20]$.

\section{Case presentation}

A 43-year old woman presented in 2011 with right upper quadrant pain. She was healthy with no significant family history of cancer. Workup with ultrasound, CT and MRI (Figure 1) revealed an enlarged right adrenal gland $(4.0 \times 3.0 \mathrm{~cm})$. She underwent an open right adrenalectomy in April 2011. There was some concern from the surgeon regarding close margins and potential rupture of the gland intra-operatively. Pathology revealed a $10.8 \times 7.5 \times 6.9$-cm lesion highly suggestive of right-sided ACC with clear margins. Postoperative restaging with 


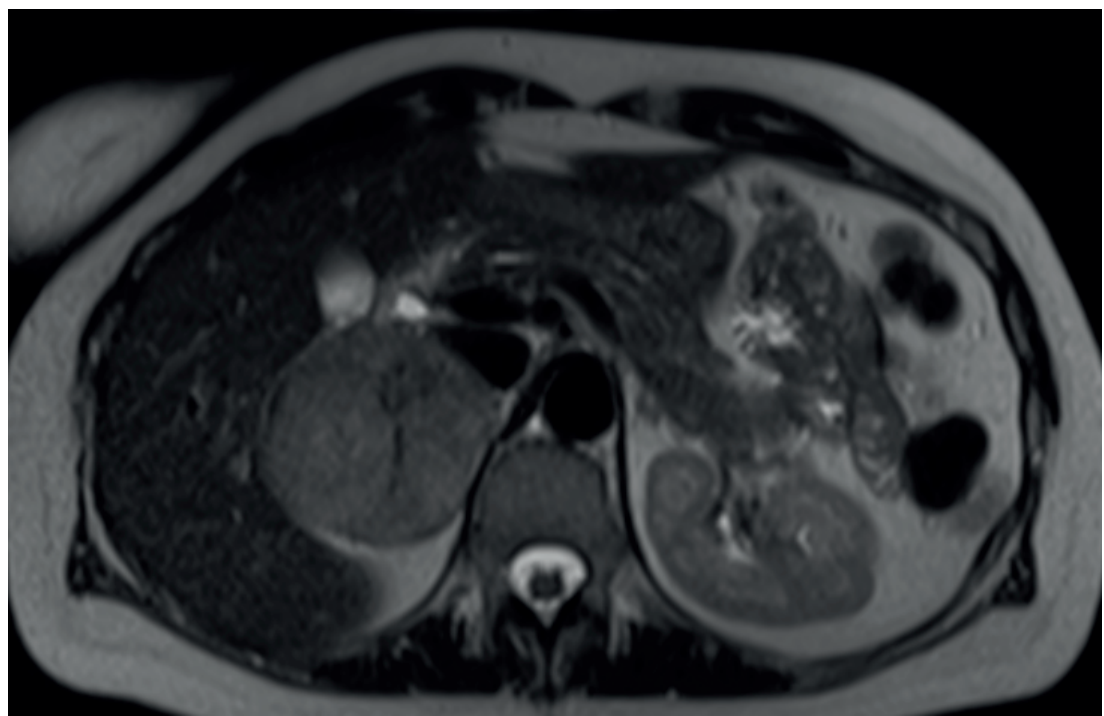

Figure 1. Axial T2 MRI imaging (2011) representing a primary right-sided adrenocortical carcinoma.
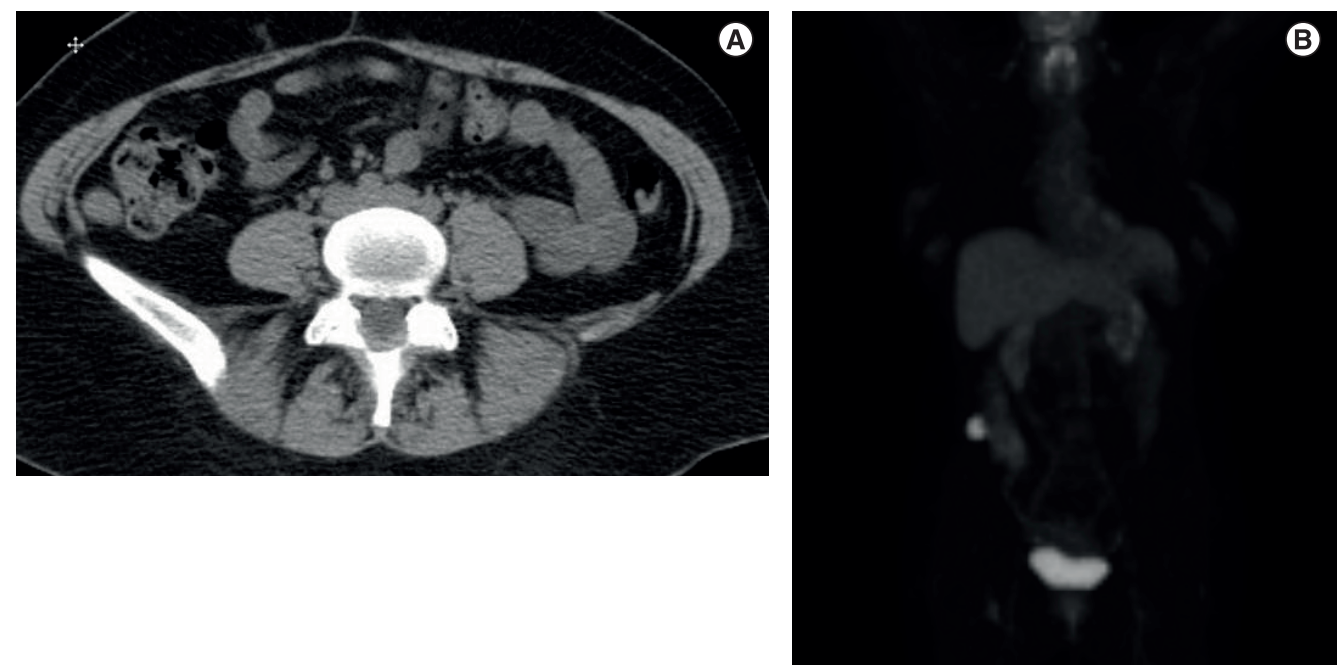

Figure 2. Evidence of adrenocortical carcinoma recurrence in the right pelvic sidewall (2017) on axial contrast CT imaging (A) and fluorodeoxyglucose PET scan (B), which displays hypermetabolic uptake in the pelvic sidewall.

CT chest, abdomen and pelvis, along with bone scan, revealed no additional sites of spread. She received adjuvant radiation therapy of $45 \mathrm{~Gy}$ in 25 fractions to the right adrenal bed and regional lymph nodes. Additionally, she received adjuvant mitotane therapy for 3.5 years after which it was discontinued due to gastrointestinal intolerance. She did not have any metabolic or endocrine symptoms.

The patient did well with no evidence of recurrence for over 5 years on CT surveillance. At 6-year follow-up, CT scan identified a nodule near the right abdominal side wall, inferior to the resected primary site and the previous radiation field (Figure 2). PET scan revealed an intensely hypermetabolic focus at the site of the nodule (Figure 2). After discussion with a multidisciplinary tumor board, the nodule was openly resected with pathology showing a $4.5 \times 3.5 \times 3.0-\mathrm{cm}$ ACC metastasis. The patient was again discussed at multidisciplinary tumor board, where it was recommended to consider adjuvant hypofractionated radiotherapy to maximize local control. She was treated adjuvantly with a dose of 22.5 Gy in 5 fractions to the tumor bed. The treatment volume included the tumor bed alone and was contoured using a preoperative CT fusion with postoperative CT simulation (Figure 3). The treatment was tolerated well with no evident toxicity and she was restarted on mitotane. Follow-up abdominal 

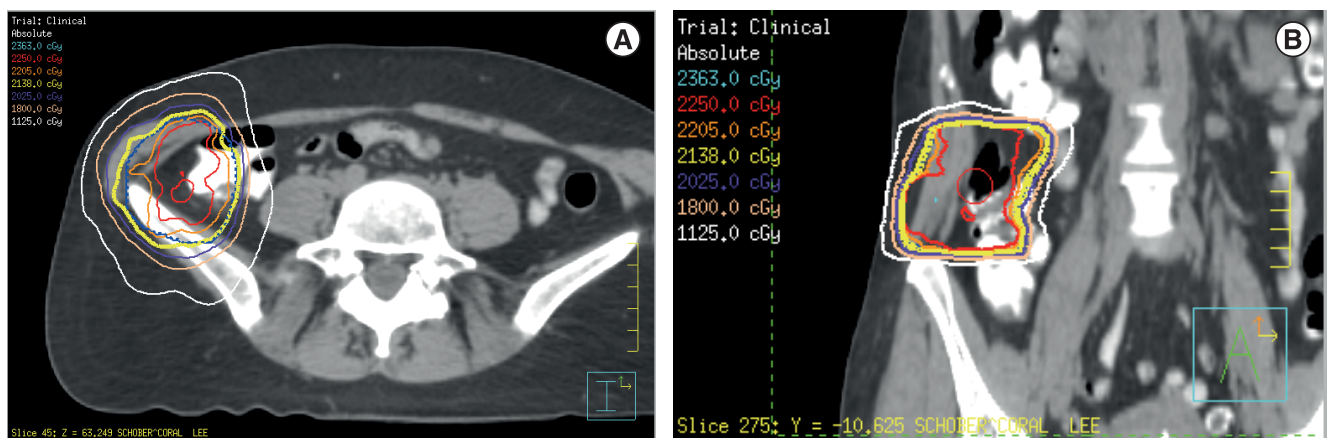

Figure 3. Coronal (A) and sagittal (B) views of the hypofractionated radiotherapy treatment plan (2017) targeting the pelvic side wall tumor bed.

imaging performed post-treatment (MR: 2 months, CT: 8, 12, 18, 24 months) revealed no evidence of local recurrence or new lesions. As of 3.25 years post-treatment, there has been no clinical evidence of recurrence.

\section{Discussion}

In summary, we present a case of a young woman initially presenting with stage 3 ACC treated with complete resection, adjuvant radiation and 3.5 years of mitotane therapy. At 6-year follow-up, she developed biopsy-proven recurrence in the pelvic side wall, immediately outside of the prior radiation field. Aggressive treatment of the isolated metastasis was pursued with complete local excision. Adjuvant hypofractionated radiotherapy was given to the resection site with limited toxicity, with no evidence of recurrence at 3.25 years post treatment. To our knowledge, this case report is the first to present an isolated ACC peritoneal metastasis treated after surgical resection with hypofractionated radiotherapy and mitotane therapy. Given the rarity of the tumor and the paucity of literature on oligometastatic management, this case may contribute to our understanding and a growing body of evidence.

Hypofractionated radiotherapy was utilized in this case for several reasons. The patient's good performance status, preferences and age, as well as the support of the tumor board, encouraged an aggressive approach. While rationale for adjuvant radiotherapy following surgical excision remains investigational, some small retrospective series have demonstrated improvements in local control with relatively modest toxicity [14,15]. Given the proximity to the prior treatment volume, a high degree of dose precision was required. Standard fractionation was offered, but the patient was very hesitant to undergo an extended course of therapy given travel time to the treatment center. A maximized safe dose was planned. Previously treated field proximity limited the delivered dose to 22.5 Gy in five fractions from the initially planned $30 \mathrm{~Gy}$ in five fractions.

Hypofractionated intensity-modulated radiotherapy may allow for targeted adjuvant therapy post-resection in recurrent or metastatic disease. This may be beneficial in improving local control without an onerous treatment course. Optimal dose, fractionation, method of delivery and treatment margins in the context of hypofractionation for adjuvant control of ACC all remain unestablished. Obtaining local control of isolated metastases remains unproven from an overall survival perspective but, when faced with young and otherwise well patients, every effort is often made to obtain long term disease control.

Hypofractionated radiotherapy has rarely been utilized to treat unresected metastatic disease. Morin et al. report a case of a 22-year old woman with a 4.4-cm liver metastasis from ACC treated with combined sorafenib and stereotactic radiation, where $42 \mathrm{~Gy}$ in six fractions was delivered to the solitary liver lesion. Over 6-month follow-up, the lesion size decreased to $2.6-\mathrm{cm}$, but disease progressed in other sites [22].

The appearance of isolated intra-abdominal drop metastasis is unique in this case. While intraoperative tumor spill has been associated with increased rates of recurrence [25], the 6-year interval from initial surgical management is unusual.

\section{Conclusion}

Adjuvant hypofractionated radiotherapy for resected, oligometastatic ACC may improve local control following surgery and can be well tolerated. Drop metastases outside previous irradiation fields can be treated with hypofractionated radiotherapy with modest toxicity, with careful attention paid to organs at risk and patient factors. Larger, 
prospective studies would be required to better understand the benefits and risks of adjuvant hypofractionated radiation in this setting. Discussion in multidisciplinary teams remains the best practice in areas of radiotherapy that lack definitive evidence.

\section{Future perspective}

Advancements in the management of ACC have thus far been complicated by the rarity of the disease, incidence of recurrent disease, heterogeneity in response to experimental treatments and therapy-associated morbidity of current approaches. We expect that the future of ACC management will include the continued development and elucidation of a diverse and robust oncology toolshed (SBRT, immunotherapy, EDP-M, HIPEC, mitotane monotherapy) with treatment strategies tailored to specific patient factors. We would hope for the development of objective clinical criteria that may help individualize multidisciplinary management for the specific presentation, recurrent versus primary disease, clinical status (including expected tolerance of treatment options) and potentially future biomarkers that may further guide therapy. Given the rarity of disease and difficulty codifying definitive treatment regimens, we expect that the management of oligometastatic ACC will be akin to treatment of a complex chronic disease - requiring close follow-up following primary resection and then highly individualized management of recurrent disease using a diverse array of therapeutic modalities.

\section{Summary points}

- Adrenocortical carcinoma (ACC) is a rare cancer of the adrenal cortex that carriers a poor prognosis and high recurrence rates.

- Salvage therapy remains poorly understood, though aggressive resection of oligometastatic ACC may be indicated in favorable patients.

- In this case report, a 43-year old woman initially presented with stage III ACC and was treated with open right adrenalectomy, adjuvant radiation therapy and 3.5 years of mitotane.

- At 6-year follow-up, she demonstrated an isolated intra-abdominal drop metastasis in the pelvic side wall. Peritoneal drop metastases are a particularly rare form of metastasis and require further documentation.

- Given the patient's young age and lack of comorbidities, this recurrence was managed with aggressive multimodal therapy including complete excision, adjuvant hypofractionated radiation and mitotane therapy.

- There was no evidence of recurrence on imaging at 3.25-year follow-up, representing a remarkable disease-free interval following salvage therapy.

- Aggressive management with adjuvant radiation and mitotane may represent an effective and well-tolerated approach for improving local control for oligometastatic ACC, including in rare cases of drop metastases.

- Larger, prospective studies would be required to better understand the benefits and risks of adjuvant therapy in this setting. Management discussion among multidisciplinary tumor boards remains best practice while definitive therapy continues to be codified.

\section{Author contributions}

Substantial contributions to the conception or design of the work; or the acquisition, analysis or interpretation of data for the work: A Magony, A Mutsaers, B Ahmad. Drafting the work or revising it critically for important intellectual content: A Magony, A Mutsaers. Final approval of the version to be published: B Ahmad. Agreement to be accountable for all aspects of the work in ensuring that questions related to the accuracy or integrity of any part of the work are appropriately investigated and resolved: $B$ Ahmad.

Financial \& competing interests disclosure

The authors have no relevant affiliations or financial involvement with any organization or entity with a financial interest in or financial conflict with the subject matter or materials discussed in the manuscript. This includes employment, consultancies, honoraria, stock ownership or options, expert testimony, grants or patents received or pending, or royalties.

No writing assistance was utilized in the production of this manuscript.

Open access

This work is licensed under the Attribution-NonCommercial-NoDerivatives 4.0 Unported License. To view a copy of this license, visit http://creativecommons.org/licenses/by-nc-nd/4.0/ 


\section{References}

1. Wong KK, Miller BS, Viglianti BL. Molecular imaging in the management of adrenocortical cancer: a systematic review. Clin. Nucl. Med. 41(8), 368-382 (2016).

2. Else T, Williams AR, Sabolch A, Jolly S, Miller BS, Hammer GD. Adjuvant therapies and patient and tumor characteristics associated with survival of adult patients with adrenocortical carcinoma. J. Clin. Endocrinol. Metab. 99(2), 455-461 (2014).

3. Stigliano A, Cerquetti L, Lardo P, Petrangeli E, Toscano V. New insights and future perspectives in the therapeutic strategy of adrenocortical carcinoma (review). Oncol. Rep. 37(3), 1301-1311 (2017).

4. Amini N, Margonis GA, Kim Y et al. Curative resection of adrenocortical carcinoma: rates and patterns of postoperative recurrence. Ann. Surg. Oncol. 23(1), 126-133 (2016).

5. Fassnacht M, Kroiss M, Allolio B. Update in adrenocortical carcinoma. J. Clin. Endocrinol. Metab. 98(12), 4551-4564 (2013).

6. Terzolo M, Angeli A, Fassnacht M et al. Adjuvant mitotane treatment for adrenocortical carcinoma. N. Engl. J. Med. 356(23), 2372-2380 (2007).

7. Srougi V, de Bassa J Jr, Tanno FY et al. Adjuvant radiotherapy for the primary treatment of adrenocortical carcinoma: are we offering the best? Int. Braz. J. Urol. 43(5), 841-848 (2017).

8. Tran TB, Maithel SK, Pawlik TM et al. Clinical score predicting long-term survival after repeat resection for recurrent adrenocortical carcinoma. J. Am. Coll. Surg. 223(6), 794-803 (2016).

9. Tiberio GAM, Ferrari V, Ballarini $\mathrm{Z}$ et al. Hyperthermic intraperitoneal chemotherapy for primary or recurrent adrenocortical carcinoma. A single center study. Cancers 12(4), 969 (2020).

10. Laganà $\mathrm{M}$, Grisanti $\mathrm{S}$, Cosentini $\mathrm{D}$ et al. Efficacy of the EDP-M scheme plus adjunctive surgery in the management of patients with advanced adrenocortical carcinoma: the Brescia Experience. Cancers 12(4), 941 (2020).

11. Glenn JA, Else T, Hughes DT et al. Longitudinal patterns of recurrence in patients with adrenocortical carcinoma. Surgery 165(1), 186-195 (2019).

12. Naing A, Meric-Bernstam F, Stephen B et al. Phase II study of pembrolizumab in patients with advanced rare cancers. J. Immunother. Cancer 8(1), e000347 (2020).

13. Luo Y, Chen SS, Zheng XG, Luo L, Wang S. The efficacy of radiation therapy in adrenocortical carcinoma: a propensity score analysis of a population-based study. Medicine (Baltimore). 96(17), 6741 (2017).

14. Fassnacht M, Hahner S, Polat B et al. Efficacy of adjuvant radiotherapy of the tumor bed on local recurrence of adrenocortical carcinoma. J. Clin. Endocrinol. Metab. 91(11), 4501-4504 (2006).

15. Habra MA, Ejaz S, Feng $L$ et al. A retrospective cohort analysis of the efficacy of adjuvant radiotherapy after primary surgical resection in patients with adrenocortical carcinoma. J. Clin. Endocrinol. Metab. 98(1), 192-197 (2013).

16. Fay AP, Elfiky A, Teló GH et al. Adrenocortical carcinoma: the management of metastatic disease. Crit. Rev. Oncol. Hematol. 92(2), 123-132 (2014).

17. Polat B, Fassnacht M, Pfreundner L et al. Radiotherapy in adrenocortical carcinoma. Cancer 115(13), 2816-2823 (2009).

18. Milgrom SA, Goodman KA. The role of radiation therapy in the management of adrenal carcinoma and adrenal metastases. J. Surg. Oncol. 106(5), 647-650 (2012).

19. Gupta N, Bansai U, Mahajan N, Yadav MS. Abdominal wall metastasis in scar after open resection of an adrenocortical carcinoma. Clin. Pract. 2(1), 9 (2012).

20. van Grevenstein WM, de Krijger RR, de Herder WW, Tilanus HW, Bonjer HJ. Abdominal wall metastasis after open resection of an adrenocortical carcinoma. Eur. J. Surg. 167(11), 871-873 (2001).

21. Bhatkule MA, Dhawle MS, Mulay VM, Bindu RS. Adrenocortical carcinoma with metastasis in abdominal wall: a rare case report. Clin. Cancer Investig. J. 4(4), 572-574 (2015).

22. Morin E, Mete O, Wasserman JD, Joshua AM, Asa SL, Ezzat S. Carney complex with adrenal cortical carcinoma. J. Clin. Endocrinol. Metab. 97(2), 202-206 (2012).

23. Foxius A, Ramboux A, Lefebvre $\mathrm{Y}$ et al. Hazards of laparoscopic adrenalectomy for Conn's adenoma. When enthusiasm turns to tragedy. Surg. Endosc. 13(7), 715-717 (1999).

24. Hamoir E, Meurisse M, Defechereux T. Is laparascopic resection of a malignant corticoadrenaloma feasible? Case report of early, diffuse and massive peritoneal recurrence after attempted laparoscopic resection. Ann. Chir. 52(4), 364-368 (1998) (Article title translated from French).

25. Miller BS, Gauger PG, Hammer GD, Doherty GM. Resection of adrenocortical carcinoma is less complete and local recurrence occurs sooner and more often after laparoscopic adrenalectomy than after open adrenalectomy. Surgery 152(6), 1150-1157 (2012). 